\title{
RDA Element Sets and RDA Value Vocabularies: Vocabularies for Resource Description in the Semantic Web
}

\author{
Fabrício Silva Assumpção ${ }^{1(\bowtie)}$, José Eduardo Santarem Segundo ${ }^{1,2}$, \\ and Plácida Leopoldina Ventura Amorim da Costa Santos ${ }^{1}$ \\ ${ }^{1}$ Graduate Program in Information Science, \\ UNESP - Universidade Estadual Paulista, Marília, Brazil \\ \{fsassumpcao,placida\} @marilia.unesp.br, santarem@usp.br \\ ${ }^{2}$ Department of Education, Information and Communication, \\ Universidade de São Paulo (USP), Ribeirão Preto, Brazil \\ santaremausp.br
}

\begin{abstract}
Considering the need for metadata standards suitable for the Semantic Web, this paper describes the RDA Element Sets and the RDA Value Vocabularies that were created from attributes and relationships defined in Resource Description and Access (RDA). First, we present the vocabularies included in RDA Element Sets: the vocabularies of classes, of properties and of properties unconstrained by FRBR entities; and then we present the RDA Value Vocabularies, which are under development. As a conclusion, we highlight that these vocabularies can be used to meet the needs of different contexts due to the unconstrained properties and to the independence of the vocabularies of properties from the vocabularies of values and vice versa.
\end{abstract}

Keywords: Resource Description and Access (RDA) - Resource Description Framework (RDF) · Functional Requirements for Bibliographic Records (FRBR) · Vocabularies

\section{Introduction}

In Information Science, the representation of resources has been based on several instruments, including metadata standards, which are created for specific contexts and focused on specific technological environments. With the Semantic Web initiative, there is an attempt to develop and implement technologies that allow the creation of descriptions of resources accessible and processable not only by its syntax, but also by its semantics.

In this sense, Information Science needs metadata standards suitable for the Semantic Web, that is, metadata standards appropriated for the creation of representations accessible by applications that use Semantic Web technologies. Based on this need, some initiatives arise in descriptive cataloging in order to create suitable metadata standards and/or to adapt those already existing. 
One of these initiatives occurs in parallel with the development and implementation of Resource Description and Access (RDA) [17] and it has as its main goal the creation of vocabularies of properties and vocabularies of values based on RDA. The results of this initiative have been released under the names RDA Element Sets and RDA Value Vocabularies.

Considering the contributions of this initiative, in this paper we aim to present the RDA Element Sets and the RDA Value Vocabularies, describing their development, classes, properties and values.

\section{RDF Data Model in Resource Description}

Descriptive cataloging deals with the description of formal aspects of information resources and establishes names and titles to provide access to these resources. In order to do it, descriptive cataloging comprises instruments for description that were created over the course of time; some of these instruments are the metadata standards and the content standards.

Over the past decades, we have faced changes in descriptive cataloging as a result of the development of information and communication technologies. Such changes require different views of the treatment of information resources and the use of practices for information organizations on the Web [2].

One of these changes involves the traditional approach that has defined catalogs' structures since the 19th century, when cataloging practices and instruments began to be formalized. The traditional catalog record, which "is composed of the values of multiple properties associated with a bibliographic entity, for example its title and physical description" [7], results from this approach.

Revision of this approach is necessary because in the Resource Description Framework (RDF) data model [16] - one of the base technologies for the Semantic Web - "the focus is on individual metadata statements represented by three-part data triples in the form subject-predicate-object" [6]. RDF is a data model that allows us to describe any kind of resources using triples composed by subject-predicate-object or, as we prefer to use in this paper, resource-property-value [2] [8].

With the focus changing, we will have individual statements, each one describing a property of the resource; for example, "The book has the title The Lord of the Rings", "The book was published in 2005" and "The book was written by J. R. R. Tolkien", rather than a single record with all the properties together, as we can see in Machine Readable Cataloging (MARC) 21 Format for Bibliographic Data. "The RDF approach is very different from the traditional library catalog record exemplified by MARC21, where descriptions of multiple aspects of a resource are bound together by a specific syntax of tags, indicators, and subfields as a single identifiable stream of data that is manipulated as a whole. In RDF, the data must be separated out into single statements that can then be processed independently from one another; processing includes the aggregation of statements into a record-based view, but is not confined to any specific record schema or source for the data. Statements or triples can be mixed and matched from many different sources to form many different kinds of user-friendly displays." [6] 